\title{
Sampling-free parametric model reduction of systems with structured parameter variation
}

Christopher Beattie, Serkan Gugercin

Department of Mathematics, Virginia Polytechnic Institute and State University, Blacksburg, USA, \{beattie, gugercin\}@vt.edu

Zoran Tomljanović

Department of Mathematics, University J.J. Strossmayer in Osijek, Osijek, Croatia, ztomljan@mathos.hr

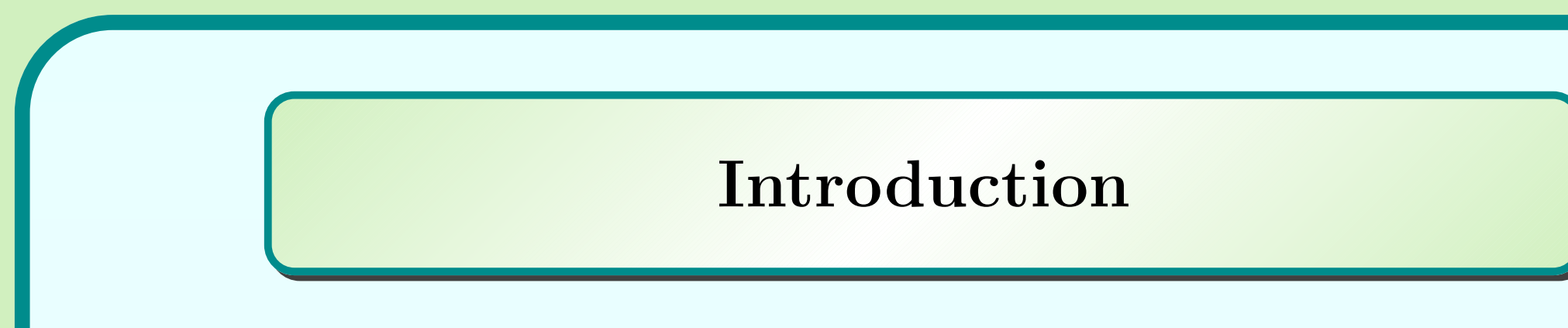

Consider a parametric LTI dynamical systems represented as

$$
E \dot{x}(t ; p)=A(p) x(t ; p)+B u(t ; p),
$$

$y(t ; p)=C x(t ; p)$,

where $E, A(p) \in \mathbb{R}^{n \times n}, B \in \mathbb{R}^{n \times m}$ and $C \in \mathbb{R}^{l \times n}$

$[E, A(p), B, C]$

- $x(t ; p) \in \mathbb{R}^{n}$ denotes the state variable

- $u(t) \in \mathbb{R}^{m}$ and $y(t ; p) \in \mathbb{R}^{l}$ represent the inputs and outputs of the system, resp.

- $A(p)$ depends on $k \ll n$ parameters $p=\left(p_{1}, p_{2}, \ldots, p_{k}\right)$ such that we may write

$$
A(p)=A_{0}+U \operatorname{diag}\left(p_{1}, p_{2}, \ldots, p_{k}\right) V^{T},
$$

where $p_{i} \geq 0$ for $i=1, \ldots, k$ and $U, V \in \mathbb{R}^{n \times k}$ are fixed. Full-order transfer function

$$
\mathbf{H}(s ; p)=C(s E-A(p))^{-1} B .
$$

\section{Approximating the full-order transfer function}

We would like to produce a reduced order model that retains the structure of parametric dependence and offers uniformly high fidelity across the full parameter range. For the full-order transfer function:

$$
\mathbf{H}(s ; p)=C\left(\widehat{A}(s)+U \operatorname{diag}\left(p_{1}, p_{2}, \ldots, p_{k}\right) V^{T}\right)^{-1} B, \quad \text { where } \widehat{A}(s)=s E-A_{0} .
$$

Most parametric model reduction methods require parametric sampling. In order to remove this need which, requires identifying particular parameter values of interest, we use the Sherman-Morrison-Woodbury formula:

$$
\mathbf{H}(s ; p)=\mathbf{H}_{1}(s)-\mathbf{H}_{2}(s) D(p)\left(I_{k}+D(p) \mathbf{H}_{3}(s) D(p)\right)^{-1} D(p) \mathbf{H}_{4}(s),
$$

where parameters are encoded in diagonal matrix $D(p)=\operatorname{diag}\left(\sqrt{p}_{1}, \sqrt{p}_{2}, \ldots, \sqrt{p}_{k}\right)$ and

$$
\begin{array}{ll}
\mathbf{H}_{1}(s)=C \widehat{A}(s)^{-1} B, & \mathbf{H}_{2}(s)=C \widehat{A}(s)^{-1} U, \\
\mathbf{H}_{3}(s)=V^{T} \widehat{A}(s)^{-1} U, & \mathbf{H}_{4}(s)=V^{T} \widehat{A}(s)^{-1} B .
\end{array}
$$

We construct a parameterized reduced order model by reducing four subsystems

$$
\left[E, A_{0}, B, C\right],\left[E, A_{0}, U, V^{T}\right],\left[E, A_{0}, U, C\right] \text {, and }\left[E, A_{0}, B, V^{T}\right],
$$

which do not depend on parameters. Additionally the first subsystem does not depend neither on matrices $U, V$.

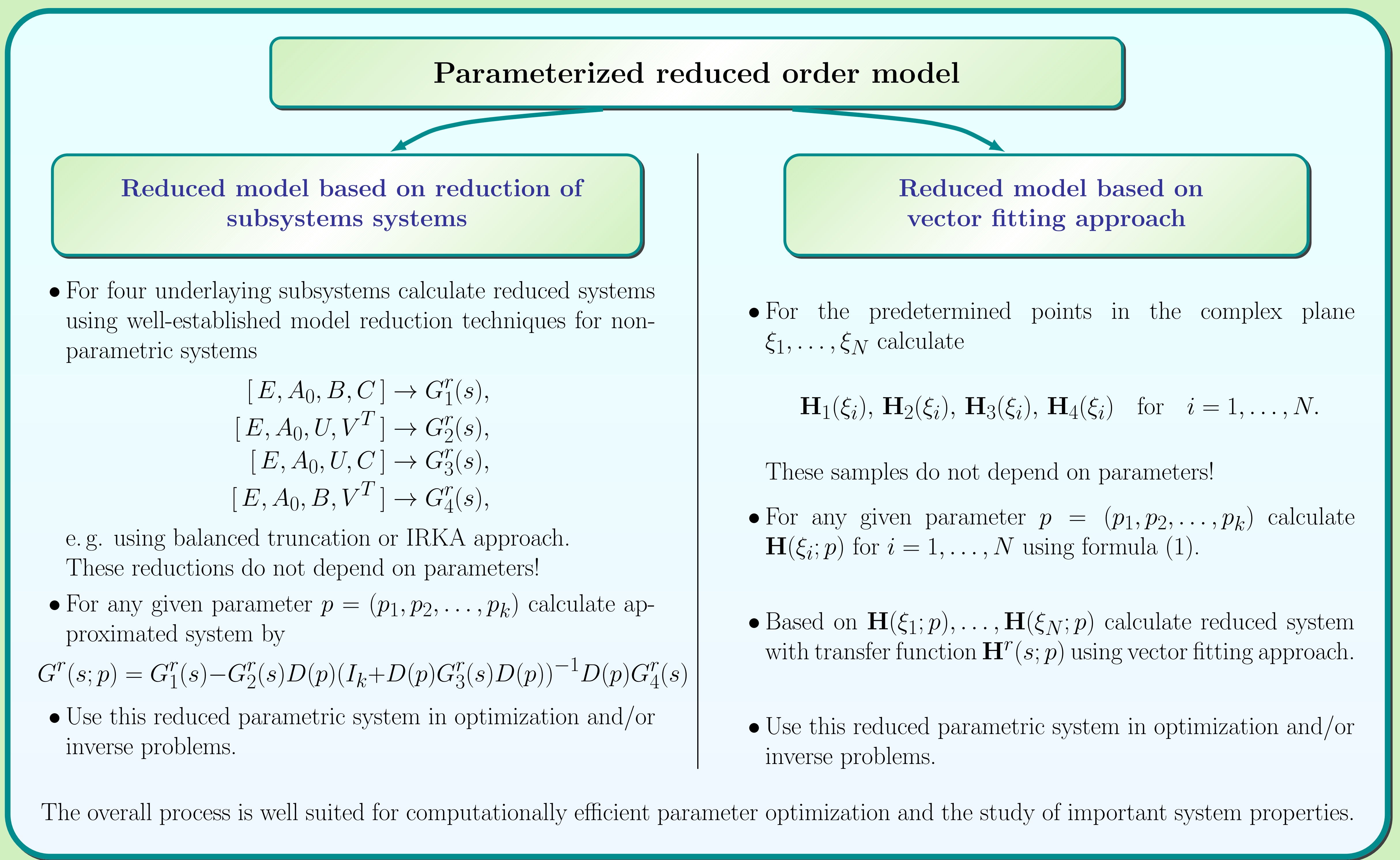

Applications to damping optimization

We consider a vibrational system described by

$M \ddot{q}(t ; p)+\left(C_{i n t}+C_{e x t}(p)\right) \dot{q}(t ; p)+K q(t ; p)=E w(t)$,

$$
z(t ; p)=H q(t ; p)
$$

- mass matrix, $M$, and stiffness matrix, $K$, are real, symmetric positivedefinite matrices of order $n$,

- $q(t ; p)$ is a vector of displacements and rotations,

- $z(t ; p)$ and $w(t)$ represent, respectively, the outputs and the inputs

(typically viewed as potentially disruptive) of the system

- damping in the structure is modeled by

$$
C_{\text {int }}+C_{\text {ext }}(p)
$$

$C_{\text {int }}$ represents internal damping,

$C_{\text {ext }}(p)=U \operatorname{diag}\left(p_{1}, p_{2}, \ldots, p_{k}\right) U^{T}$ represents external damping with gains $p_{1}, p_{2}, \ldots, p_{k}$ and $U \in \mathbb{R}^{n \times k}$ determines the placement and geometry of the external dampers.

Main problem: to determine the best damping matrix that is able to minimize influence of the disturbances, $w$, on the output of the system $z$.

Optimization criterion: $\mathcal{H}_{2}$ system norm
Consider a mass oscillator with $2 d+1$ masses and $2 d+3$ springs

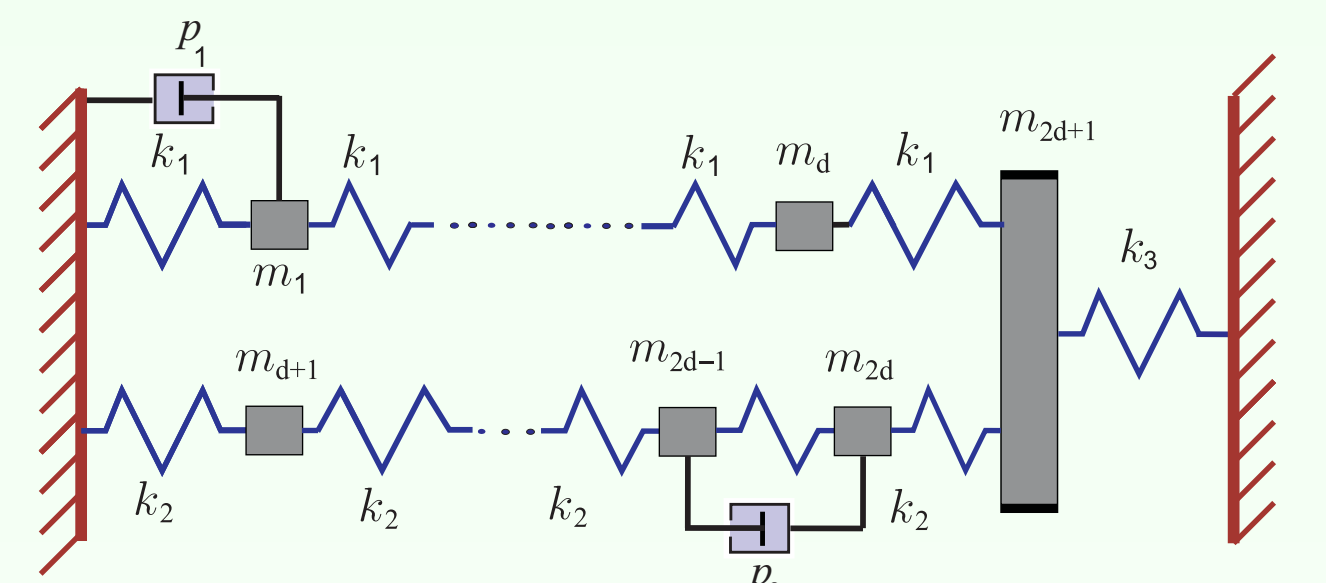

The mathematical model is given by (2) with

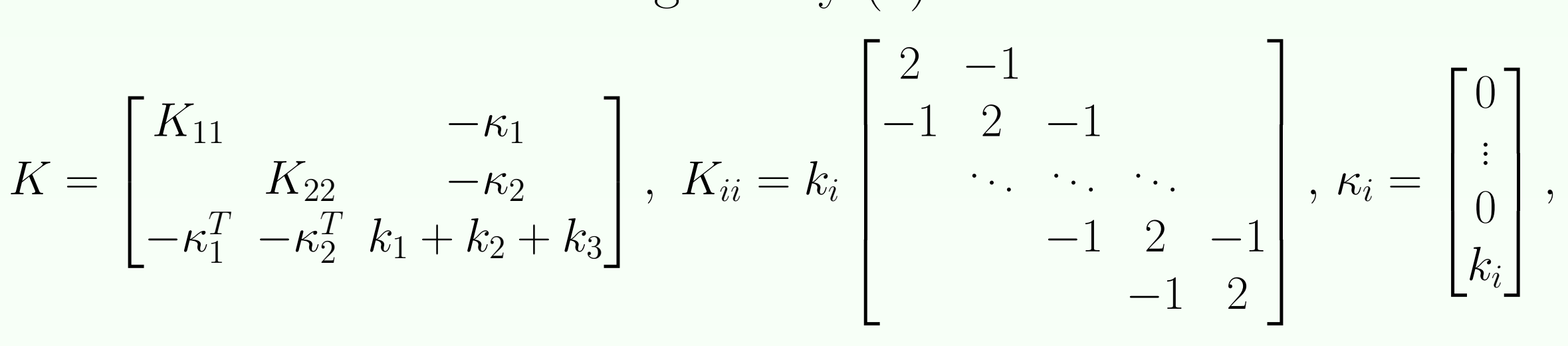

for $i=1,2$ and $M=\operatorname{diag}\left(m_{1}, m_{2}, \ldots, m_{n}\right)$.
$d=900 \Rightarrow n=1801$, with $m_{1801}=1000$ and

$$
m_{i}= \begin{cases}1000-\frac{i}{2}, & i=1, \ldots, 450 \\ i+325, & i=451, \ldots, 900 \\ 1300-\frac{i}{4}, & i=901, \ldots, n\end{cases}
$$

The stiffness values are given by

$$
k_{1}=500, k_{2}=200, k_{3}=300 .
$$

The primary excitation are 5 disturbances applied to the 4 masses closest to the left-hand side and one mass closest to the right-hand

side of oscillator.

We are interested in 2 displacements, i. e.

$$
z(t ; p)=\left[q_{400}(t ; p) q_{1300}(t ; p)\right]^{T}
$$

Internal damping is a small multiple of critical damping

$$
C_{\text {int }}=0.04 \cdot M^{1 / 2}\left(M^{-1 / 2} K M^{-1 / 2}\right)^{1 / 2} M^{1 / 2} \text {. }
$$

\section{Example}

We consider four dampers with gains $p_{1}, p_{2}, p_{3}$ and $p_{4}$ where geometry of positions is given by

$$
U=\left[e_{j_{1}}-e_{j_{1}+10}, e_{j_{2}}, e_{j_{3}}, e_{j_{3}}-e_{j_{3}+100}\right],
$$

with $j_{1} \in\{100,300,500,700\}, j_{2} \in\{150,350,550,750\}$,

$j_{3} \in\{1400,1700\} \Rightarrow 32$ different damping configurations.

Relative errors for the optimal gain

$$
10^{0}
$$

$10^{-1}$

$10^{-2}$

$10^{-3}$
$10^{-4}$

$10^{-5}$

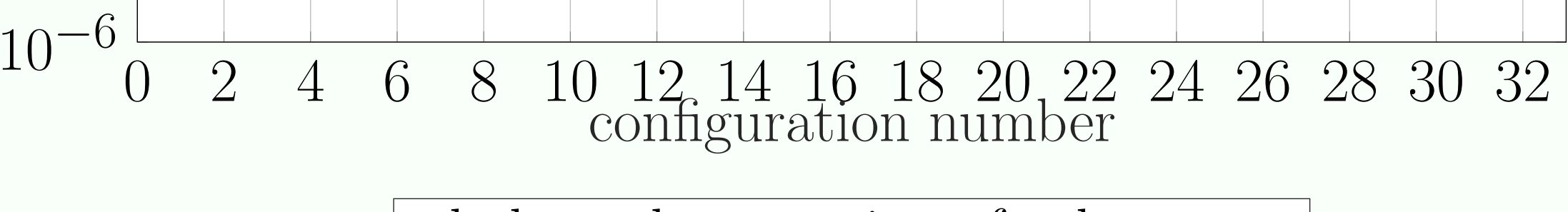

- balanced truncation of subsystems

- vector fitting

Relative errors in $\mathcal{H}_{2}$ norm at optimal gains

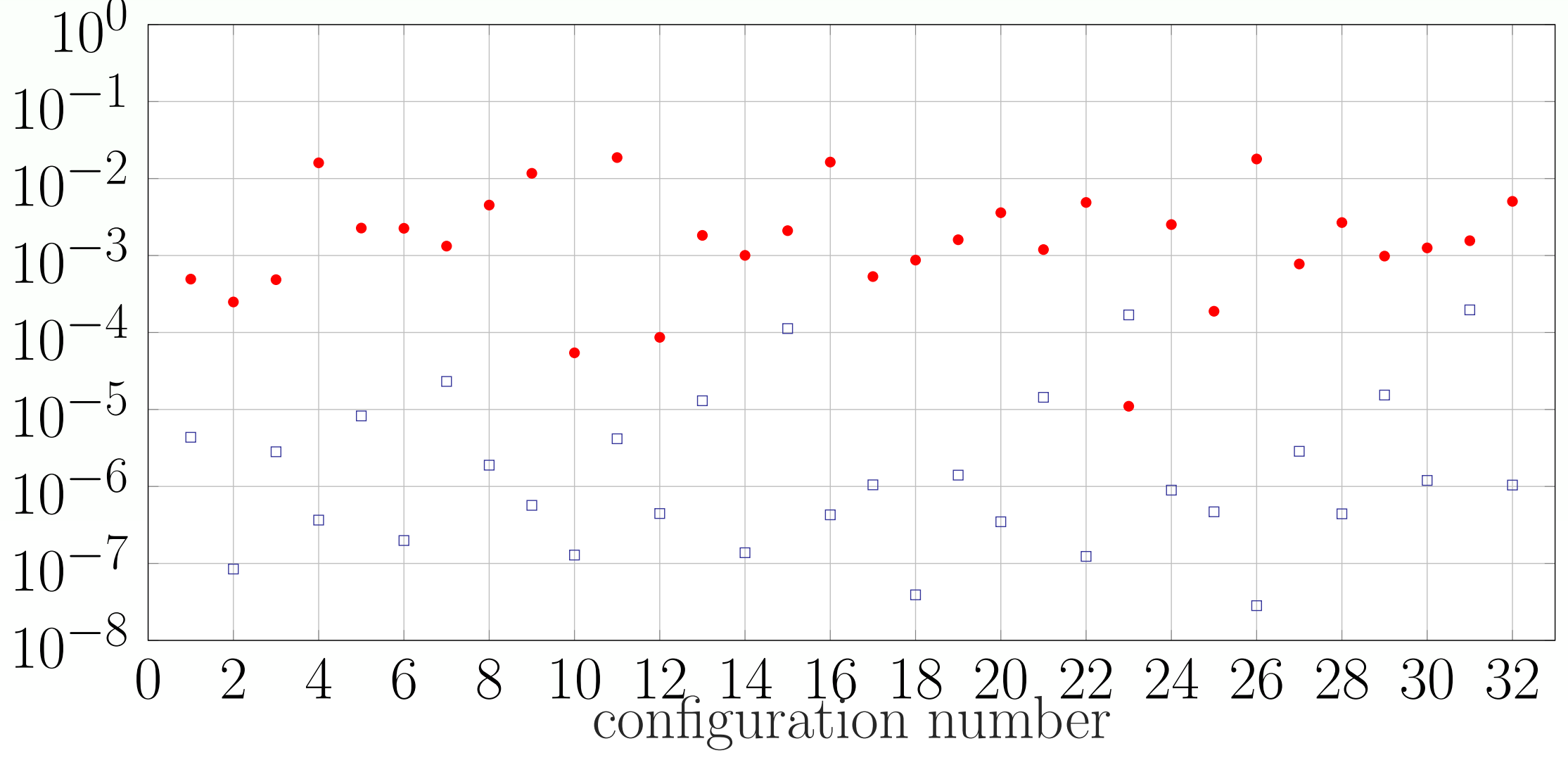

Gains were optimized with starting point $p^{0}=(100,100,100,100)$ using the full-order model and using proposed reduced systems. Relative errors in optimal values and relative errors $\mathcal{H}_{2}$ norm at optimal values values are presented.

In the approach based on balanced truncation of subsystems:

- in all damping configurations, subsystems were reduced to dimensions 500, 400, 300, 350, respectively.

In the approach based on vector fitting approach

- initial points $\xi_{i}, i=1, \ldots, N$, for $N=400$ were calculated by using dominant poles,

- order of approximation was 240

In considered parameterized reduced order models there was no need for parameter sampling.

\section{Time ratio}

In average case for one optimization of parameters, new approach was faster

$\bullet \sim 8$ times, with usage of reduced model based on balanced truncation of subsystems,

$\bullet \approx 75$ times, with usage of reduced model based on vector fitting approach

) uts 